\title{
Extracellular matrix markers for disease progression and follow-up of therapies in familial amyloid polyneuropathy V30M TTR-related
}

\author{
I. Cardoso ${ }^{\mathrm{a}}$, M. Brito ${ }^{\mathrm{a}}$ and M.J. Saraiva ${ }^{\mathrm{a}, \mathrm{b}, *}$ \\ ${ }^{a}$ Molecular Neurobiology Unit, IBMC-Instituto de Biologia Molecular e Celular, University of Porto, Porto, \\ Portugal \\ ${ }^{\mathrm{b}}$ ICBAS, University of Porto, Porto, Portugal
}

\begin{abstract}
Familial Amyloidotic Polyneuropathy (FAP) is a disorder characterized by the extracellular deposition of fibrillar Transthyretin (TTR) amyloid, with a special involvement of the peripheral nerve. Several extracellular matrix proteins have been found elevated in tissues from FAP patients, namely metalloproteinase-9 (MMP-9), neutrophil gelatinase associated lipocalin (NGAL) and biglycan. In this work we assessed the levels of MMP-9, tissue inhibitor of metalloproteinase 1 (TIMP-1), NGAL, biglycan and chondroitin sulphate (CSPG) in an FAP V30M TTR-related transgenic mouse model at different stages of TTR deposition and after two different treatment approaches to remove fibrillar deposits. Immunohistochemistry or RT-PCR analysis showed that biglycan was already increased in animals presenting TTR deposited in a non-fibrillar state, whereas MMP-9, TIMP-1, NGAL and CSPG were elevated only in mice with TTR amyloid deposits. Mice treated with doxycycline, a TTR fibril disrupter, presented lower levels of MMP-9, TIMP-1 and NGAL, suggestive of matrix recovery. Mice immunized with TTR Y78F to remove TTR deposition showed significantly lower levels of all the five tested markers, suggesting removal of fibrillar and non-fibrillar deposits. Cellular studies using oligomeric TTR showed induction of MMP-9 when compared to soluble TTR, large aggregates or fibrils. Furthermore, this induction was neutralized by an anti-receptor for advanced glycation end products (RAGE) antibody, indicating RAGE engagement in this process. Further studies in a larger number of tissue samples will indicate the application of these ECM markers in parallel with Congo Red staining in tissue characterization of pre-clinical and clinical stages in FAP and other amyloidoses.
\end{abstract}

Keywords: Extracellular matrix, familial amyloid polyneuropathy, transthyretin, metalloproteinase-9, neutrophil gelatinase associated lipocalin, biglycan

Abbreviations: FAP - Familial amyloidotic polyneuropathy; HPRT - hypoxanthine-guanine phosphoribosyl transferase; TTR transthyretin; MMP-9 - metalloproteinase 9; TIMP-1 - tissue inhibitor of metalloproteinase 1; NGAL - neutrophil gelatinase associated lipocalin; CSPG - choindroitin sulphate proteoglycan; TTR Y78F - transthyretin with a tyrosine substituted by a phenylalanine at position 78; RAGE - receptor for advanced glycation end products; RT-PCR - Reverse transcriptase-polymerase chain reaction.

\footnotetext{
* Corresponding author: Maria João Saraiva, Molecular Neurobiology, IBMC, Rua do Campo Alegre, 823, 4150-180, Porto, Portugal. Tel.: +351 22 6074900; Fax: +351 22 6099157; E-mail: mjsaraiv@ibmc.up.pt.
}

\section{Introduction}

Familial amyloid polyneuropathy (FAP) is an autosomal dominant disorder characterized by the extracellular deposition of mutated Transthyretin (TTR) amy- 
loid fibrils. It is a systemic disease which affects preferentially the peripheral nervous system and results in death in about ten years after the beginning of symptoms. FAP is characterized by early impairment of temperature and pain sensation in the feet, and autonomic dysfunction leading to paresis, malabsorption and emaciation. Amyloid deposits can occur in any part of the peripheral nervous system, including nerve trunks, plexus and sensory and autonomic ganglia.

Amyloid deposits show special tinctorial properties such as green birefringence under polarized light after staining with Congo red (CR), or staining with thioflavin $\mathrm{S}$ and $\mathrm{T}$ producing a yellow-green fluorescence. Ultrastructurally, amyloid fibrils appear as bundles of non-branched straight or coiled fibrils, 7-10 nm wide and variable length [11]; in most cases fibrils seem to be helically twisted. Non-fibrillar TTR has been identified both in vitro [6] and in vivo [27]; it was shown that asymptomatic carriers presented non-fibrillar TTR deposited in tissues for several years preceding disease. Moreover, these non-fibrillar deposits were associated with toxicity as assessed in a Schwannoma cell line by the presence of increased levels of caspase-3, oxidative stress and inflammatory markers.

In vivo amyloid formation may suffer the influence of other factors in addition to amyloidogenic structural determinants. In fact, amyloid deposits are not entirely composed of the amyloid precursor protein and several components have been found in fibrils. These include: serum amyloid P component (SAP) [21], sulphonated glycosaminoglycans (GAGs) [24], apolipoprotein E [10] and J [7], $\alpha 1$-antichymiotrypsin [1], several basement membrane components such as fibronectin, laminin and collagen type IV [23], complement proteins [22], and metal ions [3]. SAP, a decameric plasma glycoprotein, is found associated to all types of amyloid deposits [14] to which binds in a calcium-dependent manner. SAP contributes to amyloidogenesis, probably by stabilizing amyloid fibrils and retarding their clearance [20].

It has recently been shown that the ECM components metalloproteinase-9 (MMP-9), neutrophil gelatinase associated lipocalin (NGAL) and biglycan are differentially increased in tissues from V30M TTR-FAP patients [25]. MMP-9 and NGAL were found elevated in nerve and salivary gland biopsies presenting amyloid deposits but not in tissues presenting only non-fibrillar deposited TTR (FAP0 stage). In contrast, biglycan was increased earlier in disease development and thus, FAP0 tissues showed higher levels of this ECM related component. Furthermore, doxycycline was shown to act directly as a TTR fibril disrupter in vitro [5]. We also reported that doxycycline was able to disaggregate TTR amyloid deposits in vivo in transgenic mice carrying the most prevalent TTR mutation (TTR V30M) [4]. These mice recapitulate the human situation as only mice with amyloid deposits displayed increased levels and activity of MMP-9 as compared to younger animals with non-fibrillar deposits. Moreover, after doxycycline treatment, tissue MMP-9 expression and activity were decreased to levels comparable to those found in animals without TTR deposition. Other amyloidrelated components, namely SAP were also decreased, indicating fibril removal, rulling out a major effect of doxycycline on MMP-9 gene expression.

In the field of therapeutics it is important to define disease markers, not only to characterize the pathology but also to follow-up and evaluate therapeutic protocols. In this work we aimed at establishing a group of ECMrelated V30M TTR -FAP disease markers using a FAP V30M TTR-related transgenic mouse model, before and after treatments that remove amyloid deposition, namely doxycycline treatment and immunization.

\section{Material and methods}

\subsection{Animals}

TTR-V30M mice in a TTR null background (TTRV30M X TTR-KO), kindly provided by Professor Suichiro Maeda from Yamanashi University, were kept and used strictly in accordance with National and European Union guidelines for the care and handling of laboratory animals. These animals were previously analyzed in our laboratory and $\sim 60 \%$ of the animals over 1 year of age were found to have deposition as amyloid, i.e., with Congo red (CR) -positive material [26], having non-fibrillar TTR deposits at younger ages. Animals treated with doxycycline or immunized with mutant Y78F TTR, as well as the respective control groups, were previously described ([4,31], respectively) and their ages varied from 23-28 months. Animals were killed after anesthesia with ketamine/xylazine. Esophagus, stomach, small and large intestines were immediately excised and processed. For light microscopy, tissues were fixed in $4 \%$ neutral buffered formalin and embedded in paraffin. 


\subsection{Immunohistochemistry}

$5 \mu \mathrm{m}$-thick sections were deparafinated in xylol and dehydrated in a descendent alcohol series. Endogenous peroxidase activity was inhibited with $3 \%$ hydrogen peroxide $/ 100 \%$ methanol and sections were blocked in $4 \%$ bovine serum and $1 \%$ bovine serum albumin in phosphate buffered solution (PBS). Primary antibodies used were rabbit polyclonal anti-MMP-9 (Chemicon, 1:1000), rabbit polyclonal anti-tissue inhibitor of metalloproteinase 1 (TIMP-1) (Santa Cruz Biotecnhology, 1:50), rabbit polyclonal anti-biglycan (a kind gift from Dr P. Roughley, Shriners Hospitals, McGill University, Montreal, Canada, 1:500), rabbit polyclonal anti-CD14 (Santa Cruz, 1:20) and mouse monoclonal anti-chondroitin sulphate (Sigma, 1:1000), which were diluted in blocking solution and incubated overnight at $4{ }^{\circ} \mathrm{C}$. Antigen visualization was performed with the biotin-extravidin-peroxidase kit (Sigma), using 3-amino-9-ethyl carbazole (Sigma) or diaminobenzidine as substrates. Semiquantitative immunohistochemistry (SQ-IHC) analysis was done with the Universal Imaging system (NIH, Bethesda, MD, USA), which performs automated particle analysis in a measured area; that is, the area occupied by pixels corresponding to the immunohistochemical substrate's color is counted and normalized relatively to the total area. In each group, 3-6 animals were analyzed and each slide used in SQ-IHC was quantified in five different selected areas. Results shown represent \% occupied area $\pm \mathrm{SD}$.

\section{3. $R T-P C R$}

Total RNA was isolated from stomach ( $n=2$ for each treatment and controls) using Trizol (Gibco) and subjected to RT-PCR with the Superscript II kit (Invitrogen) following the manufacturer's instructions. PCR was performed for 30 cycles of denaturation at $95^{\circ} \mathrm{C}$ for $30 \mathrm{~s}$, annealing at $59^{\circ} \mathrm{C}$ for $45 \mathrm{~s}$, and polymerization at $72^{\circ} \mathrm{C}$ for $1 \mathrm{~min} 30 \mathrm{~s}$. PCR primers specific to the genes of interest were used for amplification which were designed using the PRIMER3 software (available at www.genome.wi.mit.edu) and using the sequence data from the National Centre for Biotechnology Information database; for NGAL, the sense primer was 5'-ATGTCACCTCCATCCTGGTC -3', and the antisense was 5'- CACACTCACCACCCATTCAG-3'; for HPRT, the sense primer was 5'-GTAATGATCAGTCA ACGGGGGAC-3', and the antisense was 5'-CCAGCA AGCTTGCAACCTTAACCA-3' (used to normalize the amplifications); PCR products were quantified from $1 \%$ agarose gels by densitometry using the ImageQuant 5.1 software (Molecular Dynamics). Density values of PCR products were normalized with HPRT PCR product. Results are presented as normalized density \pm SD.

\subsection{TTR production and purification}

Recombinant wild type TTR was produced in a bacterial expression system using Escherichia coli BL21 [9] and purified as previously described [2]. Protein concentration was determined using the Lowry method [17].

\subsection{Production of TTR oligomers and fibrils}

To produce TTR oligomers, the protein was dialyzed against water, $\mathrm{pH} 7.0$, and stirred for 7 days (at $0.25 \mathrm{mg} / \mathrm{ml}$ ) at room temperature [30]. For the generation of fibrils, TTR at $2 \mathrm{mg} / \mathrm{ml}$ was acidified in $0.05 \mathrm{M}$ sodium acetate $\mathrm{pH} 3.6$, for 72 hours at room temperature. Samples were then centrifuged at $15,000 \mathrm{~g}$ for 30 minutes at $4^{\circ} \mathrm{C}$, the pellet washed and resuspended in PBS and then incubated at $37^{\circ} \mathrm{C}$ for 15 days. The presence of oligomers and fibrils was confirmed by $\mathrm{Th}$ $\mathrm{T}$ binding and transmission electron microscopy.

\subsection{Cell culture and caspase-3 measurement}

RN22 cells (rat Schwannoma cell line) were propagated in $25 \mathrm{~cm}^{2}$ flasks and maintained at $37^{\circ} \mathrm{C}$ in a humidified atmosphere of $95 \%$ and $5 \% \mathrm{CO}_{2}$. Cells were grown in Dulbecco's minimal essential medium (DMEM) supplemented with $10 \%$ fetal bovine serum (FBS) (Life Technologies, Gaithersburg, MD, USA). $80 \%$ confluent RN22 cells in DMEM in $1 \%$ fetal bovine serum (FBS) were exposed for 24 hours to $2 \mu \mathrm{M}$ oligomeric TTR; alternatively, cells were previously incubated with $30 \mu \mathrm{g} / \mathrm{ml}$ of goat polyclonal anti-RAGE (receptor for advanced glycation end products) antibody (Santa Cruz) or goat non-immune IgGs (Sigma) for 3-5 hrs. Then, cells were treated with $2 \mu \mathrm{M}$ oligomeric TTR for 24 hrs; non-treated cells were used as controls. Subsequently, each flask was trypsinized and the cell pellet was lysed in $100 \mu \mathrm{L}$ hypotonic lysis buffer (Sigma) by five cycles of freeze/thawing; $40 \mu \mathrm{L}$ of each cell lysate was used in duplicate to determine caspase- 3 activation (Fluorometric caspase 3 assay kit, Sigma) following the manufacturer's instructions. The remaining cell lysate was used to measure total cellu- 
lar protein concentration using the BIO-RAD protein assay kit (Bio-Rad), using BSA as standard. Values shown are the mean of duplicates and the experiment was performed twice.

\subsection{SDS-PAGE zymography}

$10 \%$ SDS polyacrylamide gels containing gelatin (Novex Zymogram gels, Invitrogen) were used to identify MMP-9. Conditioned media $(20 \mu \mathrm{l})$ from RN22 cells incubated as described above was analyzed. Following electrophoresis, gels were incubated in Novex Zymogram renaturing buffer for $30 \mathrm{~min}$ and then overnight at $37^{\circ} \mathrm{C}$ in Novex Zymogram development buffer. Gels were then stained with $0.5 \%$ Coomassie blue in $40 \%$ methanol, $10 \%$ acetic acid for $2 \mathrm{~h}$, and destained in $40 \%$ methanol, $10 \%$ acetic acid for $1 \mathrm{~h}$. MMP-9 was identified based on molecular weight and well-established running profile on SDS-PAGE zymography.

\subsection{Statistical analyses}

All data examined were expressed as mean \pm S.E. Comparison between groups was made using the Student's t test. A p value of less than 0.05 was considered statistically significant.

\section{Results}

In a previous work we assessed the levels of MMP-9 in stomach from TTR-V30M mice [4]; only animals with TTR amyloid deposits (Congo Red (CR) positive) $(+/+)$ revealed increased amounts of MMP-9 as compared to animals presenting only non-fibrillar TTR $(+/-)$ or no deposition at all $(-/-)$. MMP-9 stained intensely in stomachs from $(+/+)$ mice, particularly at sites of amyloid deposition although co-localization was not observable most of the times. In this work, we further characterized the animal model by determining the levels of other extracellular matrix (ECM) components, such as tissue inhibitor of metalloproteinase type 1 (TIMP-1), biglycan, NGAL and CSPG, in animals with or without TTR amyloid deposition and in old animals after doxycycline treatment or immunization with TTR Y78F [31].

\subsection{MMP-9 and TIMP-1 levels}

We evaluated by IHC the levels of TIMP-1 in $(-/-)$, $(+/-)$ and $(+/+)$ mice. Our results indicated that the levels of TIMP-1 followed the trend found for MMP9 , as $(+/+)$ mice with amyloid deposition showed increased amounts of this inhibitor when compared to $(+/-)$ and $(-/-)$ animals (not shown).

Figure 1 shows the results obtained for MMP-9 and TIMP-1 before and after doxycycline or immunization treatment of mice. In both cases, it is clear that MMP-9 and TIMP-1 levels are negligible when compared to non-treated animals. The metalloproteinase was located intracellularly and staining was mainly observed in the glandular part of the stomach, although it was also possible to visualize MMP-9 in muscular areas. Since staining was either present (control mice) or absent (treated animals), we did not proceed to quantitative analysis. While MMP-9 was easily visualized by considerable intensity and distribution closely to amyloid deposits, the latter were comparatively confined to very small areas.

\subsection{Absence of inflammatory cells}

It is known that the matrix degrading enzyme MMP9 is highly expressed at sites of inflammation and contributes to the pathogenesis of various chronic inflammatory diseases. In asthma MMP-9 is upregulated and involved in remodeling processes [15,16,33]. MMP-9 also facilitates recruitment of inflammatory cells such as eosinophils and neutrophils across basement membranes [16] and in fact, inflammatory cells are a good source of MMP-9. Because doxycycline could inhibit inflammatory cell recruitment to the stomach and thus MMP-9, we investigated inflammatory cell infiltration in both control and treated mice, using an anti-CD14 antibody, a marker for macrophages and neutrophils. Inflammatory cell infiltration was not observed in either case (not shown).

\subsection{Biglycan levels}

Levels of biglycan were assessed by SQ-IHC in slides representative of $(-/-),(+/-)$ and $(+/+)$ mice as well as of doxycycline-treated and immunized animals, and results are depicted in Fig. 2. Biglycan was found overexpressed in earlier stages of deposition; thus, mice with non-fibrillar deposits $(+/-)$ presented elevated amounts of biglycan when compared to control animals $(-/-)$. Expression of biglycan 


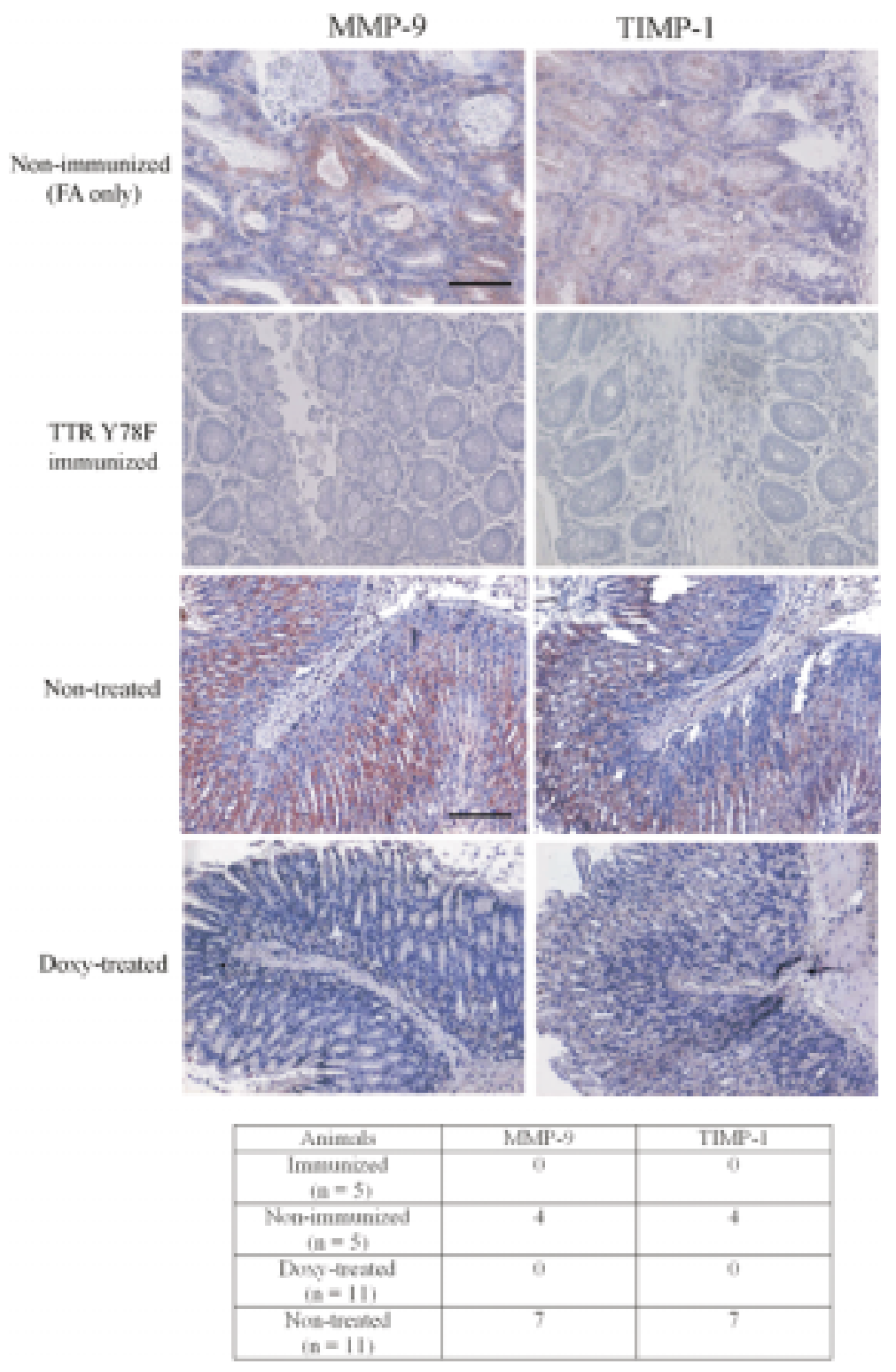

Fig. 1. Analysis of MMP-9 and TIMP-1 levels. Immunohistochemistry analysis of MMP-9 and TIMP-1 in stomach of TTR V30M mice immunized with TTR Y78F and animals that received Freud's adjuvant (FA only) (upper panels), scale bar $=200 \mu \mathrm{m}$; and in non-treated and doxycycline treated (doxy-treated) animals (lower panels), scale bar $=400 \mu \mathrm{m}$. Both immunization and doxycycline treatments resulted in negligible levels of both proteins suggesting ECM recovery. Table indicates the number of animals in each group (left column) and the number of positive cases for each ECM marker (middle and right columns).

was observed in the cytoplasm, not co-localizing with amyloid. Similar results were previously found in human V30M TTR-FAP tissues either in salivary gland and nerve biopsies. In respect to treated mice, only immunized mice showed decreased levels of biglycan (Fig. 2), indicating the treatment was efficient in removing TTR amyloid and non-fibrillar forms. By contrast, since doxycycline acts as a TTR fibril disrupter, non-fibrillar forms of TTR which are also observable in $(+/+)$ mice, persisted in tissues and thus, no changes in biglycan levels were detected in mice treated with this drug.

\subsection{Levels of CSPG}

We also compared levels of CSPG by SQ-IHC in animals of different ages, with various degrees of TTR 
Table 1

Panel of ECM markers evaluated in the V30M TTR- FAP transgenic mouse model, at different stages of disease progression, as well as the response to two different therapies. "Normal", indicates values comparable to $(-/-)$ mice and "increased" reveals levels above the $(-/-)$ animals

\begin{tabular}{lccccc}
\hline \multirow{2}{*}{ Markers } & \multicolumn{2}{c}{ Disease stage } & & \multicolumn{2}{c}{ Response to treatment } \\
\cline { 2 - 3 } \cline { 5 - 6 } & $(+/-)$ & $(+/+)$ & & Immunization & Doxycycline \\
\hline MMP-9 & & increased & & normal & normal \\
TIMP-1 & & increased & & normal & normal \\
Biglycan & increased & increased & & normal & increased \\
CSPG & & increased & & normal & increased \\
NGAL & & increased & & normal & normal \\
\hline
\end{tabular}
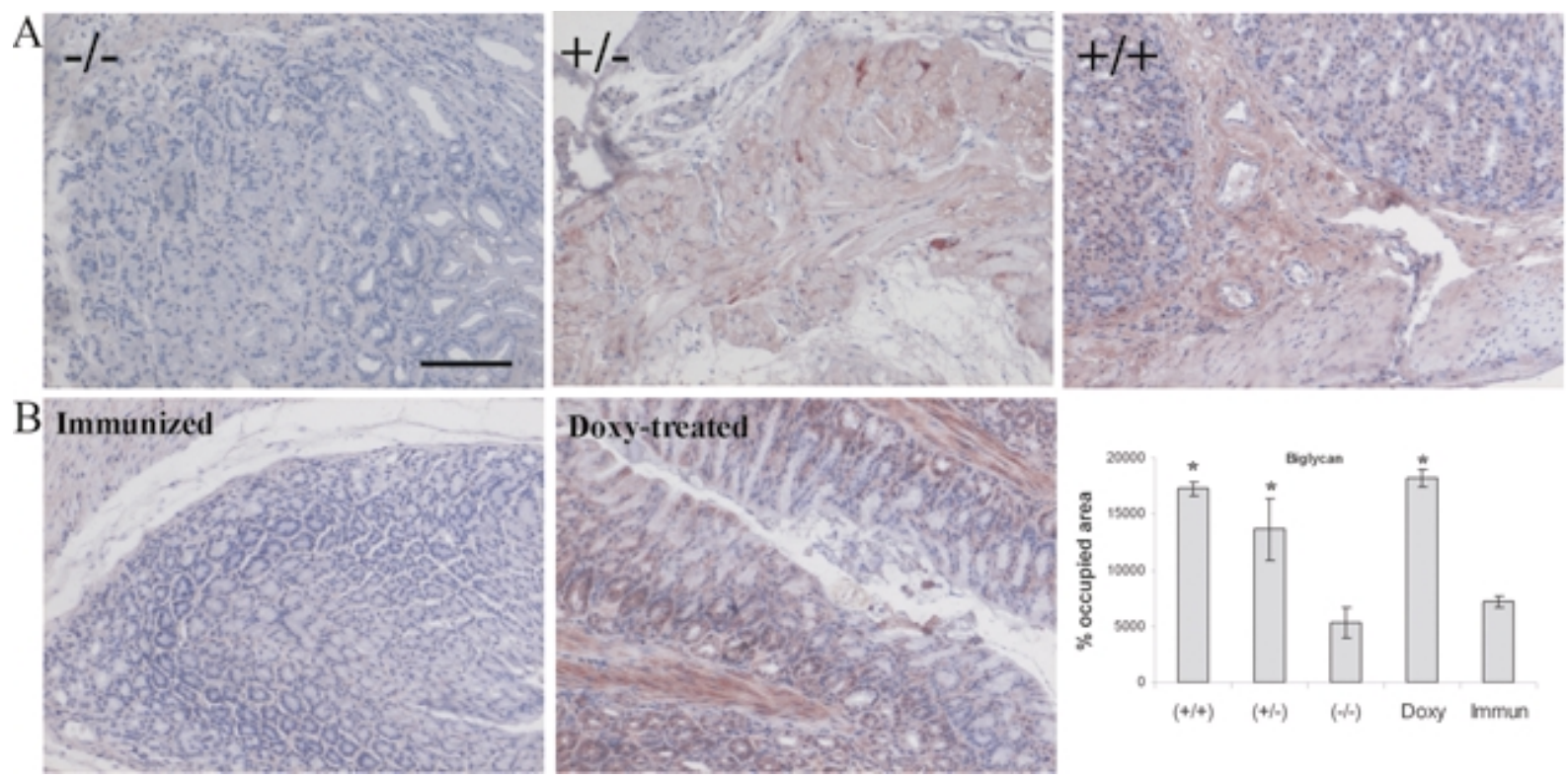

Fig. 2. Analysis of biglycan levels. A- Immunohistochemistry analysis of biglycan in stomach of TTR-V30M mice at different stages of TTR deposition $(-/-),(+/-)$ and $(+/+)$ and B- of immunized and doxycycline treated animals. Biglycan is already present in significant levels in $(+/-)$ (upper middle panel) and $(+/+)$ animals (upper right panel) when compared to control $(-/-)$ animals (upper left panel); immunization produces changes to negligible levels (lower left panel). However, doxycycline treatment resulted in biglycan levels unchanged (lower right panel). Scale bar $=400 \mu \mathrm{m}$. C- Histogram: quantification of biglycan staining in animals referred to in $\mathrm{A}$ and $\mathrm{B}{ }^{*} p<0.05$.

deposition and in mice subjected to the two above described treatments. Like MMP-9 and TIMP-1, this ECM-related component was only significantly elevated in advanced stages of deposition, i.e., in mice with amyloid deposition $(+/+)$, whereas animals showing only non-fibrillar accumulation $(+/-)$ or no deposition $(-/-)$ showed negligible amounts of CSGP (Fig. 3). These results are in agreement with the observations performed in human tissues from V30M TTR-FAP patients [19] which found CSPG significantly increased in salivary glands from V30M TTR-FAP patients in advanced stages of disease. Again, staining was cytoplasmatic and analysis of mice treated with doxycycline or by immunization revealed that only the later procedure resulted in decreased load of CSPG as compared to control non-treated animals.

\subsection{NGAL expression}

NGAL expression was investigated by RT-PCR followed by semi-quantitative PCR (Fig. 4). The results demonstrated that NGAL was elevated only in mice with amyloid deposits and thus corroborates the observations reported for human tissues [25]. Both treatments, doxycycline administration and immunization with TTR Y78F, were able to lower NGAL to normal levels as compared to non-treated controls. 

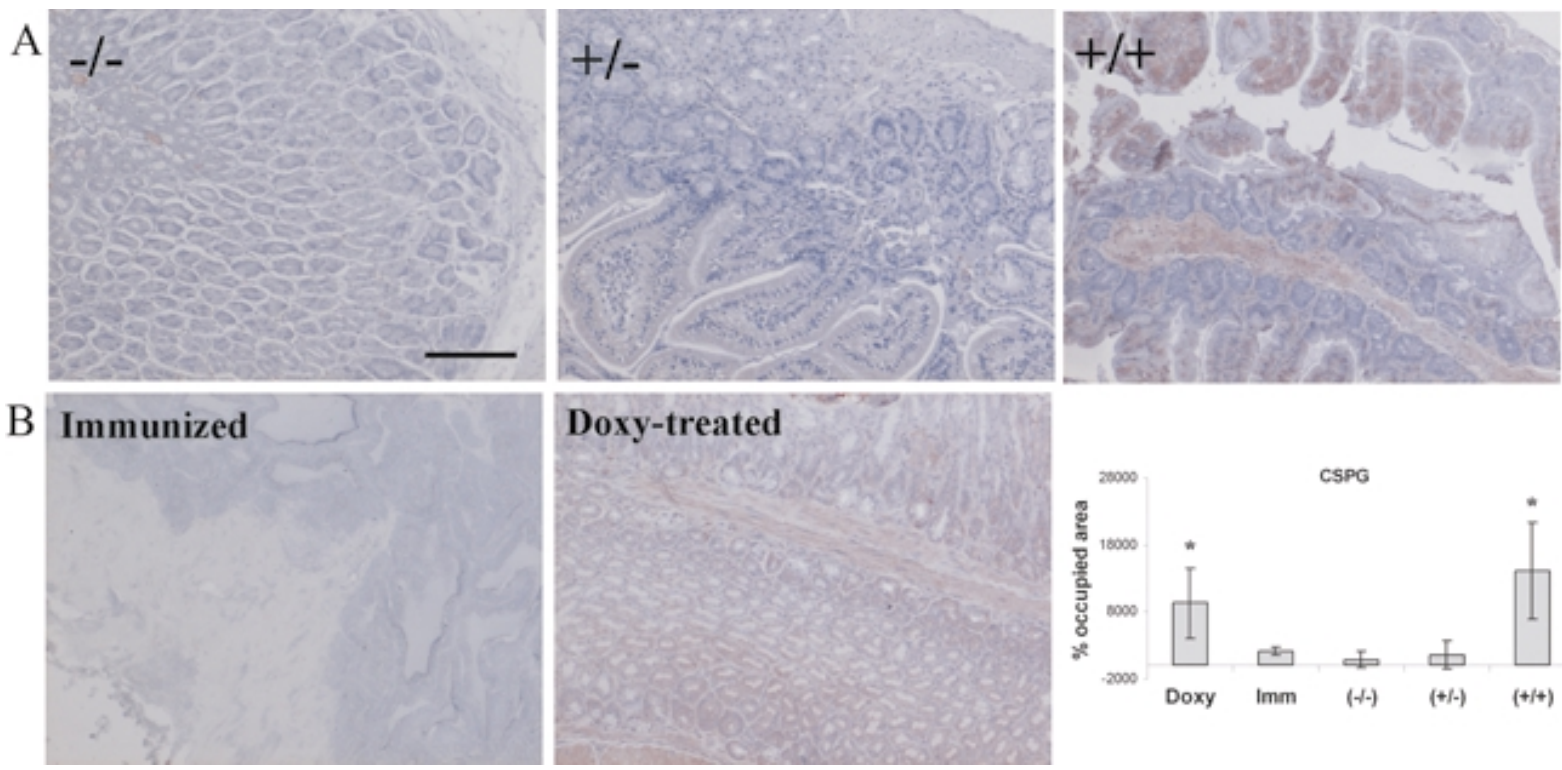

Fig. 3. Analysis of CSPG levels. A- Immunohistochemistry analysis of CSPG in stomach of TTR-V30M mice presenting different degrees of TTR deposition $(-/-),(+/-)$ and $(+/+)$, and B- immunized or doxycycline treated old mice. CSPG appears in negligible levels in animals without TTR deposition (-/-) (upper left panel) and in mice with non-fibrillar TTR $(+/-)$ (upper middle panel); in contrast, $(+/+)$ mice showed high levels of CSPG (upper right panel). While immunization returned CSPG to normal levels (lower left panel), doxycycline did not produce a significant effect (lower right panel). Scale bar $=400 \mu \mathrm{m}$. C- Histogram: quantification of CSPG staining in animals referred to in A and B. ${ }^{*} p<0.05$.

\subsection{MMP-9 induction via the receptor for advanced glycation end products (RAGE)}

We further investigated possible pathways for the activation of these selected ECM-components, in particular of MMP-9. We started by determining which TTR species trigger MMP-9 activation in vitro. Amyloid deposits in peripheral nerves occur especially in the endoneurium, where they appear close to Schwann cells and collagen fibrils [8]. We had previously established a RN22 (rat Schwann cell line) cellular assay for apoptosis assessment of TTR amyloidogenic toxic species in which apoptosis was abrogated by RAGE neutralization [28]. We used the same cellular assay to investigate MMP-9 activation by soluble, oligomeric, and fibrillar TTR; only oligomeric TTR, the very early intermediate species was able to significantly induce MMP-9 expression when compared to non-treated cells or cells treated with soluble or fibrillar TTR, as assessed by gel zymography (not shown). RAGE displays increased expression in V30M TTR-FAP tissues and its binding to TTR amyloidogenic species triggers nuclear factor (NF)- $\kappa \mathrm{B}$ expression [29]. RAGE is increased in neurons, vascular endothelium, and Schwann cells; because RAGE is also increased in the gastrointestinal tract of FAP patients [18], we used the RN22 assay to test the hypothesis of RAGE involvement in MMP9 induction by TTR oligomers as it occurs in other pathologies, such as diabetes [32]. Following blockage of the RAGE receptor, MMP-9 returned to normal levels (Fig. 5A). In parallel, caspase-3 was measured as a marker for apoptosis (Fig. 5B), revealing that the oligomeric toxic species produced caspase- 3 activation which was blocked by RAGE with concomitant MMP9 decrease.

\section{Discussion}

The process that converts a soluble protein into amyloid is complex and not fully understood. In the literature, TTR amyloid deposits were for a long time assigned as the causative agent of FAP. However, the finding that asymptomatic individuals carrying the TTR V30M mutation show a positive reaction for inflammatory markers and present non-fibrillar TTR deposits in the nerve raised the possibility that other species besides mature fibrils could be involved in the disease [27]. Thus, the search and identification of disease markers at different stages of the disease is of great importance when evaluating and characterizing disease progression and also the follow-up of therapeutic approaches. 
A

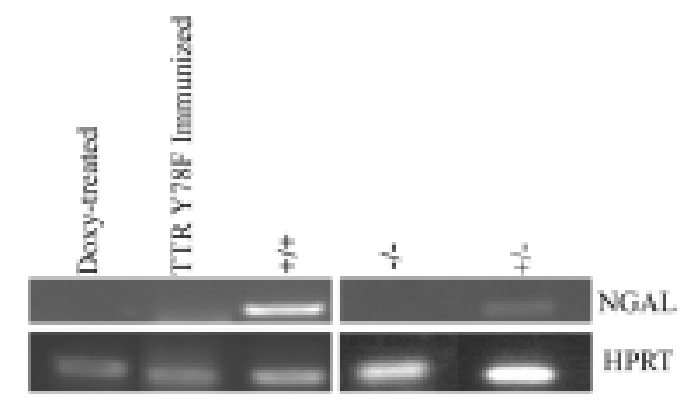

B

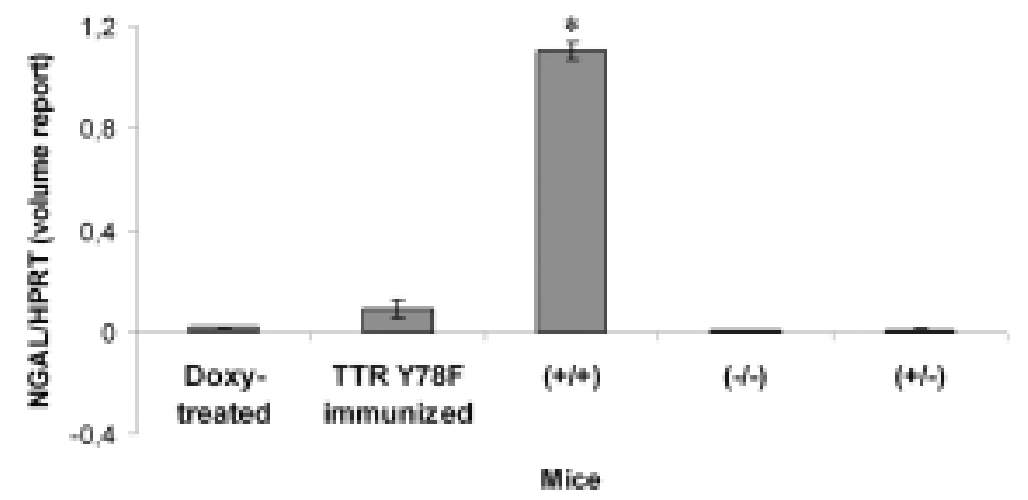

Fig. 4. Analysis of NGAL levels. RT- PCR analysis of RNA extracted from stomach of TTR-V30M mice in different stages of TTR deposition $(-/-),(+/-)$, and $(+/+)$; immunized and doxycycline treated animals. A- electrophoresis in agarose gel showing significant amplification of NGAL only for $(+/+)$ mice whereas $(+/-),(-/-)$, immunized and doxycycline treated old mice presented similar negligible levels of the NGAL gene expression. B- densitometry of NGAL/HPRT RT-PCR amplifications of animals referred to in A, revealing that NGAL is above normal levels only in $(+/+)$ mice and that both immunization and doxycycline treatment resulted in a decrease of NGAL to levels comparable to $(-/-)$ and $(+/-)$ animals. $* p<0.05$.

NGAL and biglycan were found to be elevated in biopsies of salivary glands from FAP patients compared to normal controls as assessed by microarrays analysis and semi-quantitative histochemistry [25]. MMP-9, which exists as a complex with NGAL, was also increased in FAP. Biglycan was elevated in asymptomatic individuals presenting non-fibrillar TTR deposits, whereas NGAL and MMP-9 were only increased in the presence of TTR amyloid deposits. Increased levels of CSPG were found in salivary glands from FAP patients as compared to controls [19].

Animal models represent a powerful tool to study the mechanisms underlying the pathological observations and to test therapeutic approaches. In this context, an FAP transgenic mice model was characterized [26] and found to mimic several FAP features: the presence of non-fibrillar TTR deposits $(+/-$ animals $)$ which evolved to amyloid deposition $(+/+$ animals) with aging and the association with stress markers such as nitrotyrosine [26]; MMP-9 was increased only in mice with amyloid deposition $(+/-)$ and not in animals pre- senting solely non-fibrillar deposits $(+/-)$ or no deposition $(-/-)$; SAP was also associated with amyloid deposits [4]. In the present evaluation, we further characterized the animal model by studying other ECM components. We found that TIMP-1, NGAL and CSPG were overexpressed only in mice with amyloid deposition, whereas biglycan was found elevated already in animals with non-fibrillar deposits. These observations are in agreement with the human ex vivo results. TIMP-1 was not assessed in human samples but since MMP-9 was overexpressed, it is likely that its inhibitor is also above normal levels.

The conclusions were corroborated by tissue analysis in mice treated to remove amyloid either by doxycycline or immunization protocols $[4,31]$; the results obtained in immunized mice indicated a decrease of all five markers under study, whereas doxycycline treated animals showed improvement only in MMP-9, TIMP-1 and NGAL, as levels of biglycan and CSPG remained unaltered. We could not detect any inflammatory cell infiltration in control animals (a common feature to 
A

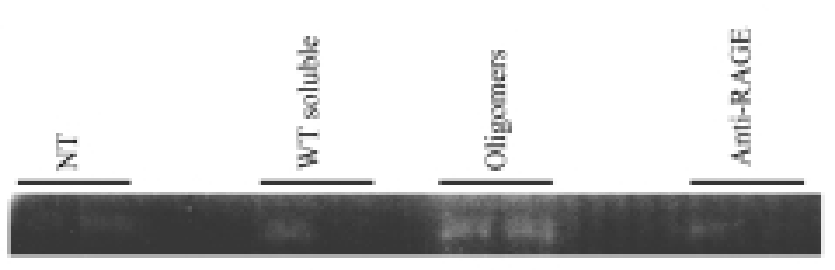

MMP-9 activity

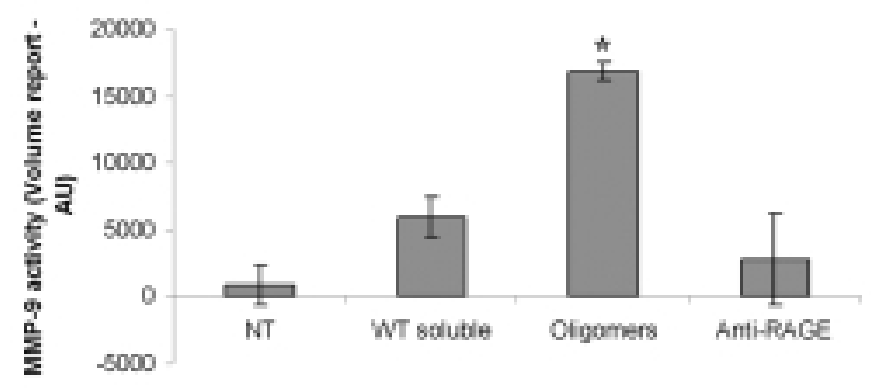

B

Caspase-3 activation

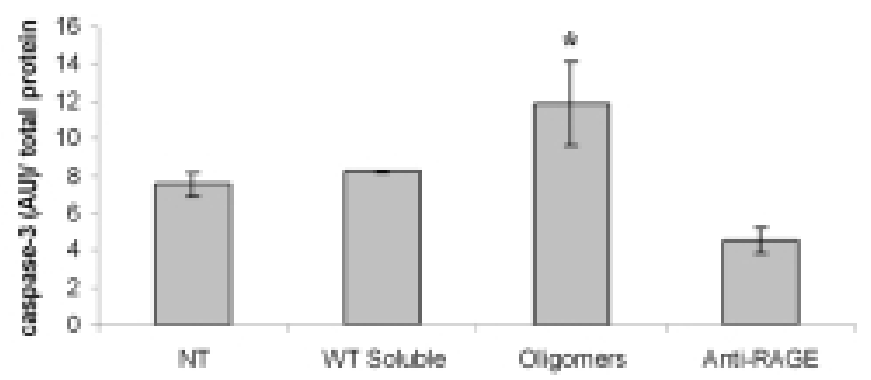

Fig. 5. Assessment of RAGE involvement in MMP-9 activation. A: zymogram of conditioned media from non-treated or cells treated with TTR different species: soluble, oligomeric, or pre-incubated with an anti-RAGE specific antibody. Histogram: densitometry of MMP-9 activity in cells treated as described above, showing activation of MMP-9 by oligomeric TTR but not by the soluble counterpart or in non-treated cells; Blockage of the RAGE receptor abolished MMP-9 activation. ${ }^{*} p<0.06$. B: Activation of caspase- 3 in RN22 cells exposed for 24 hours to $1 \mu \mathrm{M}$ of the different TTR species referred to in A. Significant caspase-3 activation was observed in the presence of TTR oligomeric species; toxicity of TTR oligomers was prevented by pre-incubating the cells with anti-RAGE antibody. $* p<0.05$.

FAP patients) and thus, decreased MMP-9 and TIMP-1 levels in doxycycline-treated mice were not due to inhibition of inflammation by doxycycline. Furthermore, at least in vitro, doxycycline acts directly on TTR amyloid fibrils, disaggregating them [5]. The decrease in MMP-9 related to amyloid removal and not a consequence of inhibition by doxycycline is also supported by our findings in tissues from mice subjected to an immunization protocol: MMP-9 was also negligible in the later procedure.

Since biglycan is elevated in the presence of nonfibrillar TTR deposits and doxycycline is a TTR fibril disrupter and not an inhibitor, elevated biglycan levels can be attributed to non-fibrillar aggregates present in tissues of doxycycline treated mice.

As for CSPG, since immunization leads to the com- 
plete removal of TTR deposits (both fibrillar and nonfibrillar) whereas doxycycline administration only disaggregates fibrillar deposits, TTR aggregates deriving from fibril disruption could account for the presence of CSPG, as this component is part of amyloid fibrils as previously described by Inoue and colleagues [13]. In contrast, MMP-9, TIMP-1 and NGAL are not part of the fibril, but their expression responds to the presence of fibrillar material. Under the conditions of the study, we can also conclude that immunization is more efficient than doxycycline treatment in removing TTR deposition.

In summary, we characterized the distribution of several ECM markers in our animal model; we suggest MMP-9 and CSPG as complementary to CR evaluation to determine the presence of amyloid, since MMP-9 and CSPG staining are easily assessed in opposition to congophilic material which might be scarce and not easily identified in samples (human or from animal models). Further studies in a larger number of tissue samples will indicate the application of these ECM markers in parallel with Congo Red staining in tissue characterization of pre-clinical and clinical stages in FAP and other amyloidoses.

Zymographic analysis of MMP-9 indicated that in a cellular system, only oligomeric TTR is capable of inducing significant MMP-9 activation. The increase was at both protein and gene expression (not shown) levels. MMP-9 activity was decreased when RAGE was blocked, suggesting RAGE engagement in MMP9 activation in FAP. The relation RAGE/MMP-9 has been previously suggested in other diseases such as diabetes [32] and atherosclerosis [12].

The fact that in the cellular system MMP-9 increased in the presence of TTR oligomeric species whereas in vivo significant levels of this metalloproteinase are only visible in amyloid laden tissues is indicative that other factors play a role in vivo. It is possible that:

1. Other molecules involved in the mechanism of amyloid formation and/or ECM regulation inhibit MMP-9 up-regulation in the early disease stages

2. As the disease evolves and the system collapses, with concomitant amyloid deposition, deregulation occurs and MMP-9 is overexpressed.

3. Other components of amyloid in FAP tissues not present in vitro, directly or indirectly, are responsible for the observed MMP-9 up-regulation in amyloid laden tissues.

Taken together, the ECM components we have studied adequately correlate with amyloid deposition and future studies should address the molecular bases of such correlation.

\section{Acknowledgments}

We thank Tânia Ribeiro for animal handling, Rossana Correia for tissue processing and Paul Moreira for protein production and purification. This work was supported by grants (POCTI and POCI programs) from Foundation for Science and Technology (FCT), and Gulbenkian Foundation, Portugal, and by a FCT fellowship to I.C. (SFRH / BPD / 9416 / 2002).

\section{References}

[1] C.R. Abraham, D.J. Selkoe and H. Potter, Immunochemical identification of the serine protease inhibitor alpha 1antichymotrypsin in the brain amyloid deposits of Alzheimer's disease, Cell 52 (1988), 487-501.

[2] M.R. Almeida, A.M. Damas, M.C. Lans, A. Brower and M.J. Saraiva, Thyroxine binding to transthyretin Met119. Comparative studies of different heterozygotic carriers and structural analysis, Endocrine 6 (1997), 309-315.

[3] A.I. Bush, W.H. Pettingell, G. Multhaup, M. d Paradis, J.P. Vonsattel, J.F. Gusella, K. Beyreuther, C.L. Masters and R.E. Tanzi, Rapid induction of Alzheimer A beta amyloid formation by zinc, Science 265 (1994), 1464-1467.

[4] I. Cardoso and M.J. Saraiva, Doxycycline disrupts transthyretin amyloid: evidence from studies in a FAP transgenic mice model, FASEB J 20 (2006), 234-239.

[5] I. Cardoso, G. Merlini and M.J. Saraiva, 4'-iodo-4'Deoxydoxorubicin and tetracyclines disrupt transthyretin amyloid fibrils in vitro producing non- cytotoxic species. Screening for TTR fibril disrupters, FASEB J 17 (2003), 803809.

[6] I. Cardoso, C.S. Goldsbury, S.A. Müller, V. Olivieri, S. Wirtz, A.M. Damas, U. Aebi and M.J. Saraiva, Transthyretin fibrillogenesis entails the assembly of monomers: A molecular model for in vitro assembled transthyretin amyloid-like fibrils, $J$ Mol Biol 317 (2002), 687-699.

[7] N.H. Choi-Miura, Y. Ihara, K. Fukuchi, M. Takeda, Y. Nakano, T. Tobe and M. Tomita, SP-40,40 is a constituent of Alzheimer's amyloid, Acta Neuropathol (Berl) 83 (1992), 260-264.

[8] A. Coimbra and C. Andrade, Familial amyloid polyneuropathy: an electron microscope study of peripheral nerve in five cases. I. Interstitial changes, Brain 94 (1971), 199-206.

[9] H. Furuya, M.J.M. Saraiva, M.A. Gawinowicz, I.L. Alves, P.P. Costa, H. Sasaki, I. Goto and Y. Sakaki, Production of recombinant human transthyretin with biological activities toward the understanding of the molecular basis of familial amyloidotic polyneuropathy (FAP), Biochemistry 30 (1991), 2415-2421.

[10] G. Gallo, T. Wisniewski, N.H. Choi-Miura, J. Ghiso and B. Frangione, Potential role of apolipoprotein-E in fibrillogenesis, Am J Pathol 145 (1994), 526-530.

[11] G.G. Glenner, Amyloid deposits and amyloidosis. The betafibrilloses (first of two parts), N Engl J Med 302 (1980), 12831292.

[12] M.A. Hofmann, E. Lalla, Y. Lu, M.R. Gleason, B.M. Wolf, N. Tanji, L.J. Ferran Jr., B. Kohl, V. Rao, W. Kisiel, D.M. Stern and A.M. Schmidt, Hyperhomocysteinemia enhances vascular inflammation and accelerates atherosclerosis in a murine model, J Clin Invest 107 (2001), 675-683. 
[13] S. Inoue, M. Kuroiwa, M.J. Saraiva, A. Guimarães and R. Kisilevsky, Ultrastructure of familial amyloid polyneuropathy amyloid fibrils: examination with high-resolution electron microscopy, J Struct Biol 124 (1998), 1-12.

[14] S. Inoue and R. Kisilevsky, Beta-amyloid fibrils of Alzheimer's disease: pathologically altered, basement membrane-associated microfibrils? Ital J Anat Embryol 106 (2001), 93-102.

[15] E.A. Kelly and N.N. Jarjour, Role of matrix metalloproteinases in asthma, Curr Opin Pulm Med 9 (2003), 28-33.

[16] Y.C. Lee, H.B. Lee, Y.K. Rhee and C.H. Song, The involvement of matrix metalloproteinase-9 in airway inflammation of patients with acute asthma, Clin Exp Allergy 31 (2001), 1623-1630.

[17] O.H. Lowry, N.J. Rosenbrough, A.L. Farr and J. Randall, Protein measurement with the Folin phenol reagent, $J$ Biol Chem 193 (1951), 265-275.

[18] N. Matsunaga, I. IAnan, S. Forsgren, R. Nagai, P. Rosenberg, S. Horiuchi, Y. Ando and O.L. Suhr, Advanced glycation end products (AGE) and the receptor for AGE are present in gastrointestinal tract of familial amyloidotic polyneuropathy patients but do not induce NF- $\kappa \mathrm{B}$ activation, Acta Neuropathol 104 (2002), 441-444.

[19] F.A. Monteiro, PhD thesis. Porto University, 2006.

[20] M.B. Pepys, Pathogenesis, diagnosis and treatment of systemic amyloidosis, Philos Trans R Soc Lond B Biol Sci 356 (2001), 203-210; discussion 210-211. Review.

[21] M.B. Pepys, Amyloidosis: some recent developments, $Q J$ Med 252 (1988), 283-298. Review.

[22] J. Rogers, N.R. Cooper, S. Webster, J. Schultz, P.L. McGeer, S.D. Styren, W.H. Civin, L. Brachova, B. Bradt, P. Ward et al., Complement activation by beta-amyloid in Alzheimer disease, Proc Natl Acad Sci USA 89 (1992), 10016-10020.

[23] D.L. Scott, G. Marhaug and G. Husby, Comparative studies of the high molecular weight amyloid fibril proteins and similar components from normal tissues, Clin Exp Immunol 52 (1983), 693-701.

[24] A.D. Snow, J. Willmer and R. Kisilevsky, Sulfated glycosaminoglycans: a common constituent of all amyloids? Lab Invest 56 (1987), 120-123.
[25] M.M. Sousa, J.B. do Amaral, A. Guimaraes and M.J. Saraiva, Up-regulation of the extracellular matrix remodeling genes, biglycan, neutrophil gelatinase-associated lipocalin, and matrix metalloproteinase-9 in familial amyloid polyneuropathy, FASEB J 19 (2005), 124-126.

[26] M.M. Sousa, R. Fernandes, J.A. Palha, A. Taboada, P. Vieira and M.J. Saraiva, Evidence for early cytotoxic aggregates in transgenic mice for human transthyretin, Am J Pathol 161 (2002), 1935-1948.

[27] M.M. Sousa, I. Cardoso, R. Fernandes, A. Guimaraes and M.J. Saraiva, Deposition of transthyretin in early stages of familial amyloidotic polyneuropathy: evidence for toxicity of nonfibrillar aggregates, Am J Pathol 159 (2001), 1993-2000.

[28] M.M. Sousa, S. Du Yan, R. Fernandes, A. Guimaraes, D. Stern and M.J. Saraiva, Familial amyloid polyneuropathy: receptor for advanced glycation end products-dependent triggering of neuronal inflammatory and apoptotic pathways, J Neurosci 21 (2001), 7576-7586.

[29] M.M. Sousa, S.D. Yan, D. Stern and M.J. Saraiva, Interaction of the receptor for advanced glycation end products (RAGE) with transthyretin triggers nuclear transcription factor $\mathrm{kB}(\mathrm{NF}-$ _B) activation, Lab Invest 80 (2000), 1101-1110.

[30] P.F. Teixeira, F. Cerca, S.D. Santos and M.J. Saraiva, Endoplasmic reticulum stress associated with extracellular aggregates. Evidence from transthyretin deposition in familial amyloid polyneuropathy, J Biol Chem 281 (2006), 21998-22003.

[31] H. Terazaki, Y. Ando, R. Fernandes, K. Yamamura, S. Maeda and M.J. Saraiva, Immunization in familial amyloidotic polyneuropathy: counteracting deposition by immunization with an Y78F TTR mutant, Lab Invest 86 (2006), 23-31.

[32] T. Wendt, E. Harja, L. Bucciarelli, W. Qu, Y. Lu, L.L. Rong, D.G. Jenkins, G. Stein, A.M. Schmidt and S.F. Yan, RAGE modulates vascular inflammation and atherosclerosis in a murine model of type 2 diabetes, Atherosclerosis $\mathbf{1 8 5}$ (2006), 70-77.

[33] S.E. Wenzel, S. Balzar, M. Cundall and H.W. Chu, Subepithelial basement membrane immunoreactivity for matrix metalloproteinase 9: association with asthma severity, neutrophilic inflammation, and wound repair, J Allergy Clin Immunol 111 (2003), 1345-1352. 


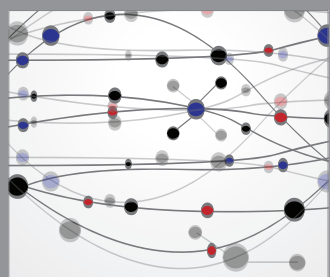

The Scientific World Journal
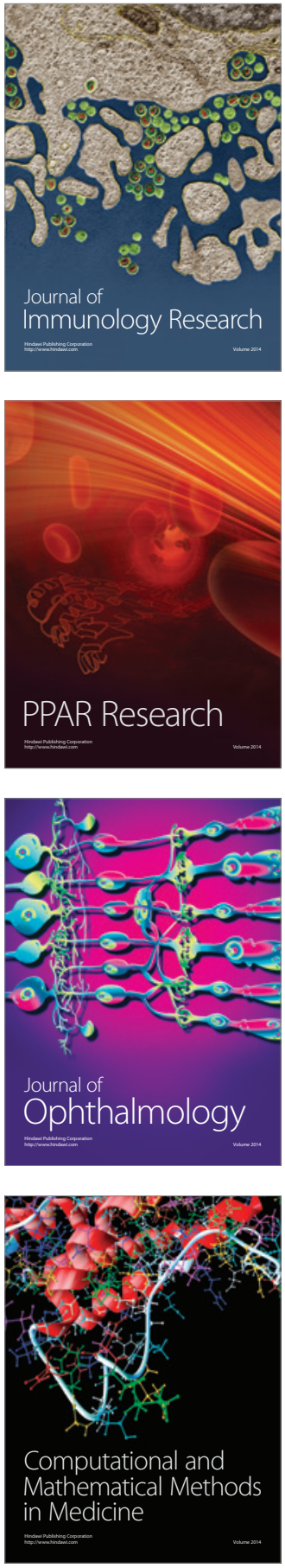

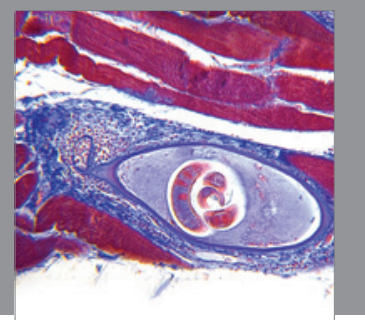

Gastroenterology

Research and Practice
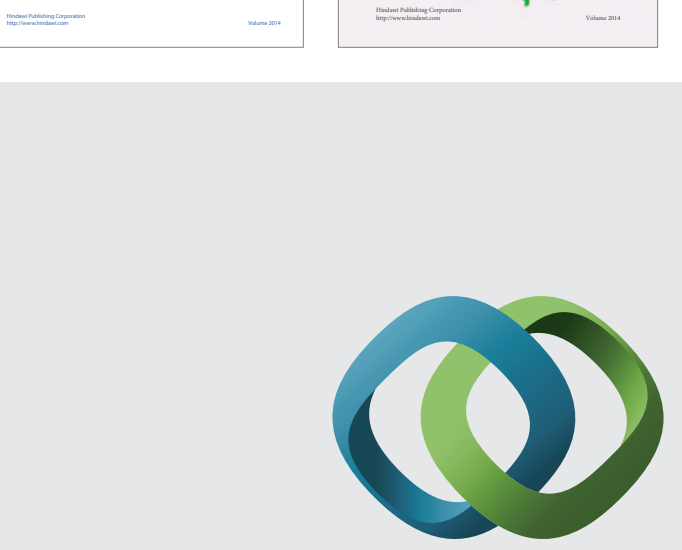

\section{Hindawi}

Submit your manuscripts at

http://www.hindawi.com
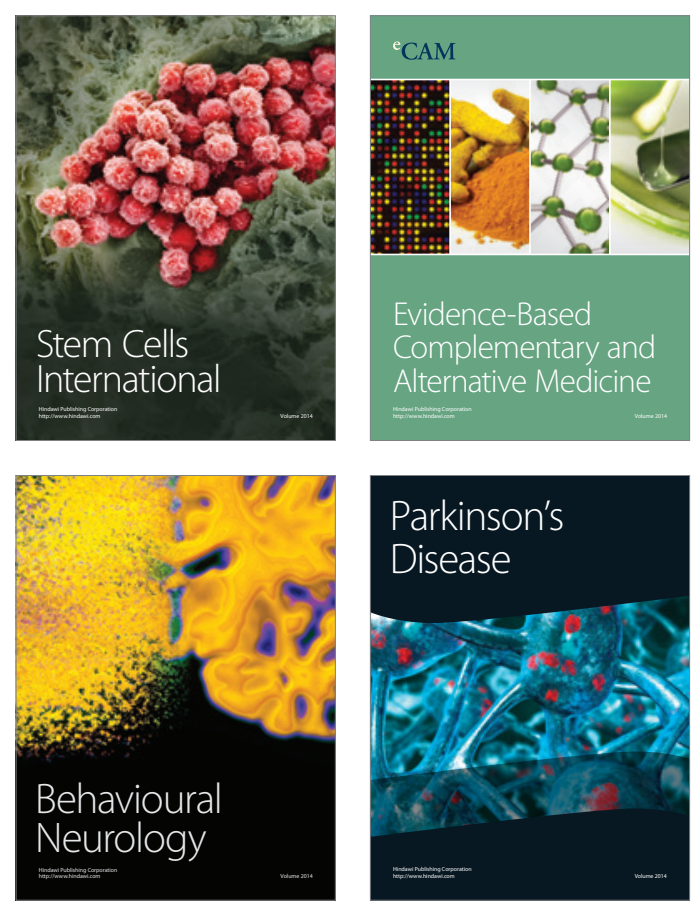

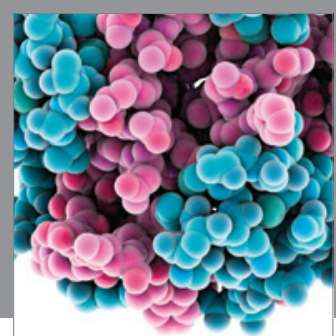

Journal of
Diabetes Research

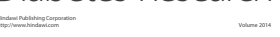

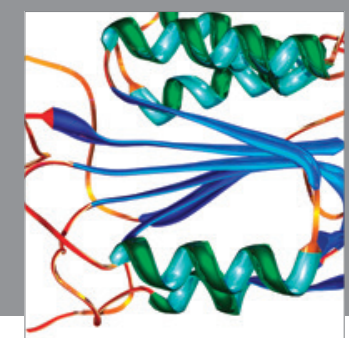

Disease Markers
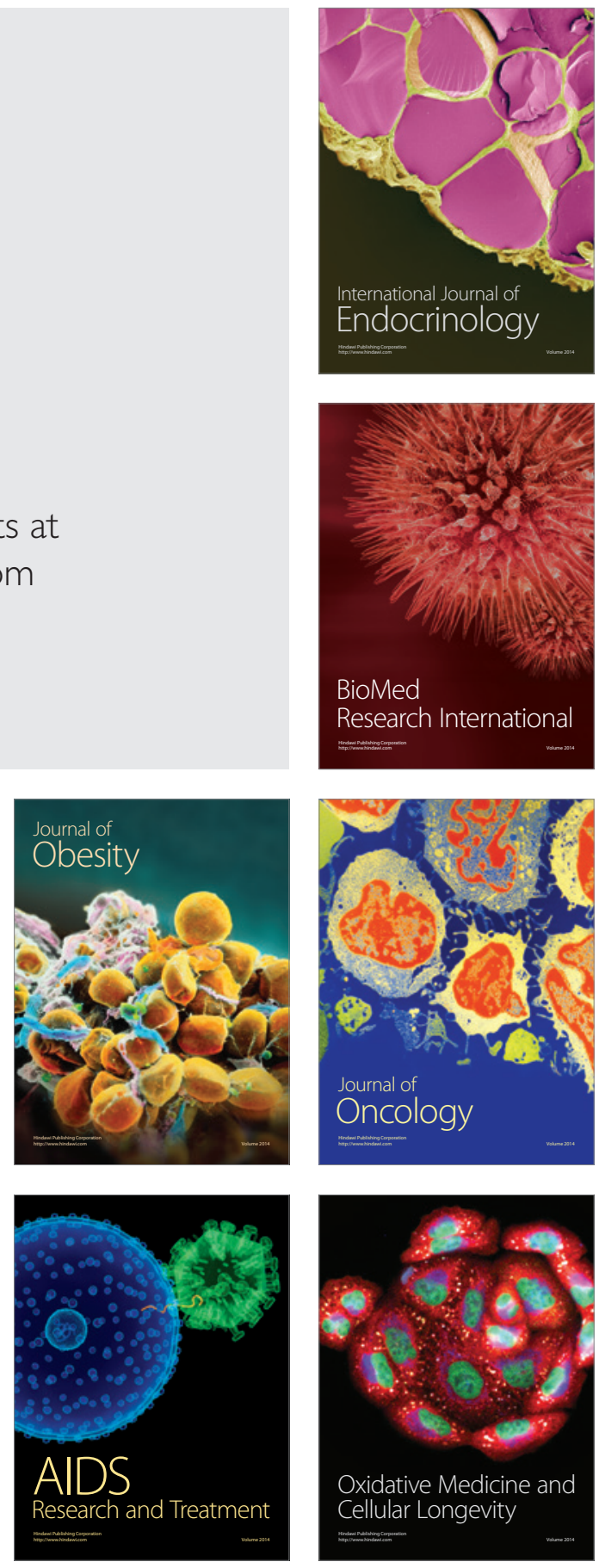